\title{
SPD-Fraktion und Reformpolitik: Wie viel Mitsteuerung war möglich bei der Vorbereitung der Agenda 2010?
}

\author{
Karlheinz Niclauß
}

Parlamentarische Regierungssysteme entsprechen nicht dem traditionellen Gewaltenteilungsmodell, das Exekutive, Legislative und Gerichtsbarkeit nebeneinander stellt. An die Stelle der von Montesquieu abgeleiteten Dreiteilung tritt hier die Konkurrenz zwischen Regierungsmehrheit und Opposition: Die Spitzenpositionen der Exekutive und die Parlamentsmehrheit bilden eine politische Einheit, während die nicht an der Regierung beteiligten Fraktionen die Aufgabe der öffentlichen Kritik und Kontrolle übernehmen. In Wirklichkeit ist das Regierungslager jedoch nicht so einheitlich, wie das Modell vorgibt. Im Falle einer Koalitionsregierung wird die Differenzierung von vornherein deutlich sichtbar. Zwischen der parlamentarischen Regierung und ihren Parlamentsfraktionen besteht ebenfalls ein Spannungsverhältnis, das durch die zunehmende Komplexität politischer Entscheidungen und den zunehmenden Einfluss der Medien verstärkt wird.

Auf Seiten der Regierung führt diese Entwicklung zu einer Aufwertung des Regierungschefs (Premierminister, Ministerpräsident). Mit Hilfe eines eigenen Arbeitsstabes kann er in der Regel die Aktivitäten seiner Ressortminister überwachen und bei seinen politischen Projekten auf den Sachverstand in den Ministerien zurückgreifen. In der Bundesrepublik war dieser administrative Rückhalt einer der Gründe für die Entstehung der Kanzlerdemokratie. Weitere Merkmale dieses Regierungstyps liegen im Parteiensystem: der deutliche Gegensatz zwischen Regierungs- und Oppositionslager sowie die Führungsrolle des Bundeskanzlers in der größten Regierungspartei. Hinzu kommen das außenpolitische Engagement des Kanzlers (oder der Kanzlerin) und die Fokussierung der Medien auf die Person des Regierungschefs. Die Verfassungsbestimmungen einschließlich der vielzitierten Richtlinienkompetenz haben im Vergleich hierzu eine untergeordnete Bedeutung ${ }^{1}$.

\section{Parlamentarische Mitsteuerung?}

Der Einfluss der Regierungsfraktionen nahm im parlamentarischen Regierungssystem ebenfalls zu. Eine großzügige Finanzierung aus öffentlichen Mitteln ermöglichte ihnen (und der Opposition) den Aufbau eines umfangreichen Mitarbeiterstabs und die Professionalisierung ihrer Arbeitsweise. Hinzu kommt der große Einfluss der Fraktionsmitglieder

1 Vgl. Karlheinz Niclauß, Kanzlerdemokratie. Regierungsführung von Konrad Adenauer bis Gerhard Schröder, Paderborn 2004, S. 67 - 100; Karl-Rudolf Korte / Manuel Fröhlich, Politik und Regieren in Deutschland, Paderborn 2004, S. 79 - 91; Ludger Helms (Hrsg.), Institutions and Institutional Change in the Federal Republic of Germany, London / New York 2000, S. 32 ff., S. $65 \mathrm{ff}$. 
auf die politische Willensbildung in den Parteien. Nahezu alle Bundestagsabgeordneten nehmen in ihren Parteien Funktionen auf Bundes-, Landes- oder kommunalpolitischer Ebene wahr. Beides zusammen, der Ausbau der Hilfsdienste und die personelle Verflechtung, haben zur Folge, dass die Parteienforschung bereits von „Fraktionsparteien“ spricht ${ }^{2}$.

Regierungsfraktionen können aufgrund ihrer engen Verbindung zur „Basis“ der Parteien und Wähler wie ein Vorwarnsystem wirken. Sie sollen eine „bürgerferne“ Regierungstätigkeit verhindern, die durch Verluste bei Umfragen und Wahlen sanktioniert wird ${ }^{3}$. Da sie für die Gesetzgebung zuständig sind, kontrollieren sie gleichzeitig ihre Regierung. Diese Kontrolle ist allerdings in der Regel nicht öffentlich, weil offene Kritik ein negatives Medienecho für die eigene Führungsmannschaft hervorrufen würde. Nach der Studie von Manfred Schwarzmeier machen Fraktionen und einzelne Abgeordnete ihren Einfluss überwiegend auf informellen Wegen geltend. Ihre Interventionen wirken sich in der Regel positiv für das betreffende Projekt aus und verbessern die „Performance“ der Regierung. Der Deutsche Bundestag ist in erster Linie Arbeitsparlament und berät in seine Ausschüssen und Fraktionsarbeitskreisen die Gesetzesvorlagen und Haushaltsentwürfe bis ins Detail. Obwohl die Gesetzgebung in der Regel in den Referaten und Abteilungen der Ministerien vorbereitet wird, haben die Regierungsfraktionen die Möglichkeiten zur parlamentarischen Mitsteuerung ${ }^{4}$. Aus Sicht der Parlamentarier gilt für den gesamten Bereich der Gesetzgebung das „Struck'sche Gesetz“, demzufolge keine Vorlage so aus dem Bundestag hinausgeht, wie sie hineingekommen ist ${ }^{5}$. Eine andere Frage ist, ob die Abgeordneten der Regierungsfraktionen in der Lage sind, die so genannte Richtungskontrolle auszuüben. Hierunter ist die Aufsicht über das Regierungshandeln im Hinblick auf die Chancen bei der nächsten Bundestagswahl oder bei anstehenden Landtagswahlen zu verstehen. Zu prüfen, ob die Regierungsinitiativen mit den programmatischen Grundlinien ihrer Partei übereinstimmen, gehört ebenfalls zur Aufgabe der Regierungsfraktionen.

\section{Der Weg zur Agenda 2010}

Die Weigerung der Bundesregierung, an der Intervention des Iraks teilzunehmen, war in der Regierungszeit Gerhard Schröders zweifellos die spektakulärste außenpolitische Entscheidung. In der Innenpolitik nimmt die als „Agenda 2010“ bezeichnete Regierungserklärung diesen Rang ein. Sie trug zum Scheitern der rot-grünen Koalition bei und beeinflusst bis zur Gegenwart die politischen Debatten in Deutschland. Deswegen liegt es nahe, die Frage nach der parlamentarischen Mitwirkung der größten Regierungsfraktion an dieser Richtungsentscheidung zu stellen. Hatte die SPD-Fraktion überhaupt Einfluss auf die Agenda-Rede vom 14. März 2003? War sie zumindest informiert über den Inhalt? Das

2 Vgl. Eckhard Nickig, Von der Mitglieder- zur Fraktionspartei: Abschied von einer Fiktion, in: ZParl, 30. Jg. (1999), H. 2, S. 382 - 389; Karlheinz Niclauß, Das Parteiensystem der Bundesrepublik Deutschland, Paderborn 2002, S. 251 - 259; Peter Lösche, Zustand und Perspektiven der SPD, in: Hans Zehetmair (Hrsg.), Das Deutsche Parteiensystem, Wiesbaden 2004, S. $104-116$.

3 Vgl. Suzanne S. Schüttemeyer, Fraktionen im Deutschen Bundestag 1949-1997, Opladen / Wiesbaden 1998, S. 36 f., S. 312 ff.

4 Vgl. Manfred Schwarzmeier, Parlamentarische Mitsteuerung. Strukturen und Prozesse informalen Einflusses im Deutschen Bundestag, Wiesbaden 2001, S. 304 - 308.

5 Vgl. Peter Struck, So läuft das. Politik mit Ecken und Kanten, Berlin 2010, S. 46 f. 
wichtigste Dokument aus der Vorbereitungsphase der Agenda ist ein Papier des Kanzleramts mit dem Titel „Auf dem Weg zu mehr Wachstum, Beschäftigung und Gerechtigkeit“. Dieses Papier wurde im Auftrag von Kanzleramtschef Frank-Walter Steinmeier vom Leiter des Planungsstabes, Heiko Geue, in Kooperation mit den Staatssekretären der Fachministerien verfasst. Es diente als Vorlage für eine Planungsklausur, die am 5. Dezember $2002 \mathrm{im}$ Kanzleramt stattfand ${ }^{6}$. Die These, das Papier sei dem CSU-Abgeordneten Horst Seehofer zugespielt worden, während die SPD-Abgeordneten es „nicht zu Gesicht" bekamen, lässt sich kaum aufrecht erhalten, denn der Text wurde unmittelbar nach der Formulierung in der Fraktion bekannt ${ }^{7}$. Bereits am 20. Dezember führte Schröder ein Gespräch mit Vertretern der „Parlamentarischen Linken“ und bat sie, ihre Kommentierung des Papiers schriftlich zu formulieren. Die von den Abgeordneten Michael Müller, Angelica Schwall-Düren und Gernot Erler verfasste Antwort ging dem Bundeskanzler am 20. Dezember zu ${ }^{8}$.

Der Entschluss Schröders, das zunächst unverbindliche Papier aus dem Kanzleramt zur Grundlage einer Regierungserklärung zu machen, ergab sich aus den Wahlresultaten und Umfrageergebnissen: Während die SPD bei der Bundestagswahl vom 22. September 2002 gleichauf mit den Unionsparteien lag, zeigten die Meinungsumfragen in den darauf folgenden Wochen ein anderes Bild: Die Zustimmungskurve der CDU/CSU stieg steil an, die der Sozialdemokraten sank deutlich. Im Dezember 2002 notierte die Forschungsgruppe Wahlen bei ihrer Frage nach der politischen Stimmung 26 Prozent für die SPD und 55 Prozent für die CDU/CSU. Die Medien warfen der Koalition und dem Kanzler mangelnde Reformfähigkeit vor. Die ersten Landtagswahlen nach der Bundestagswahl in Hessen und Niedersachsen am 2. Februar 2003 wurden angesichts dieser Vorgaben mit Spannung erwartet. Ihre Ergebnisse signalisierten für die Sozialdemokraten höchste Alarmstufe: In Niedersachsen wurde am 2. Februar ihre Alleinregierung abgewählt und durch eine CDU/ FDP-Regierung unter Christian Wulff ersetzt. Die SPD verlor 14,5 Prozentpunkte, während die Grünen konstant blieben. In Hessen erreichte der regierende Ministerpräsident Roland Koch (CDU) am selben Tag eine absolute Mehrheit und konnte ohne seinen bisherigen Koalitionspartner FDP weiterregieren. Die SPD verlor hier mehr als zehn Punkte, während die Grünen fast drei hinzu gewannen.

Im Bundesrat konnten die von der CDU/CSU und der FDP regierten Länder aufgrund dieser Resultate ihre bis dahin knappe Mehrheit ausbauen und verfügten nun über 41 von 69 Stimmen. Ein rot-grüner Wahlsieg in beiden Bundesländern hätte der Bundesregierung noch die Möglichkeit eröffnet, das Abstimmungsverhältnis in der Länderkammer mit Hilfe einer der Großen Koalitionen in Bremen oder Brandenburg in ihrem Sinne „umzudrehen“. Franz Müntefering wies außerdem in der ersten Sitzung des Geschäftsführenden Fraktionsvorstandes nach den beiden Landtagswahlen darauf hin, dass jetzt im Vermittlungsausschuss Stimmengleichheit zwischen Regierungs- und Oppositionslager bestehe. Deswegen müsse man bereits im Gesetzgebungsverfahren des Bundestages Absprachen mit der Oppo-

6 Vgl. leicht gekürzte Fassung, in: FAZ vom 4. Januar 2003, S. 5, ausführliche Version als PDFDatei unter www.ak-sozialpolitik.de (Abruf am 26. Oktober 2010).

7 So Karl-Rudolf Korte / Manuel Fröhlich, a.a.O. (Fn. 1), S. 297.

8 Schreiben von Gernot Erler / Michael Müller / Angelica Schwall-Düren an Bundeskanzler Gerhard Schröder vom 3. Januar 2003 (dem Autor freundlicherweise von Dr. Angelica Schwall-Düren zur Verfügung gestellt). 
sition treffen ${ }^{9}$. Die Wahlergebnisse in Hessen und Niedersachsen wirkten sich auch auf die Stimmung in der Fraktion aus. Während Abgeordnete bis dahin Einzelmaßnahmen und die unzureichende Information kritisierten, wurden nun auch grundsätzliche Bedenken laut. Symptomatisch ist eine Kontroverse zwischen Schröder und Hans-Ulrich Klose über die Irak-Politik der Bundesregierung, die in der nächsten Spiegel-Ausgabe nahezu wörtlich wiedergegeben wurde ${ }^{10}$.

Zugleich gingen im Kanzleramt die Vorbereitungen für die „große Rede“ voran. Wenn man dem Bericht des „Spiegels“ glauben kann, fand am 13. Februar ein wichtiges Treffen in der Katholischen Akademie Berlin statt, an dem neben der Mannschaft des Kanzleramts Schröders Redenschreiber Reinhard Hesse teilnahm ${ }^{11}$. Bei der Terminplanung spielten drei Gesichtspunkte eine Rolle: ein Brief des FDP-Vorsitzenden Wolfgang Gerhard vom 5. Februar, auf den Schröder in irgendeiner Form antworten musste; der bevorstehende Einmarsch der USA in den Irak, dem der Kanzler zuvorkommen wollte, und die Beratung der Steuergesetze im Bundesrat am 14. März. Der dort zu erwartenden Kritik an der Bundesregierung wollte Schröder mit seiner Offensive entgegentreten. Der technokratische Titel „Agenda 2010“ stammt angeblich von der Frau des Bundeskanzlers. Schröder hatte ursprünglich „Neue Chancen für Deutschland“"vorgesehen ${ }^{12}$.

Die spätere Diskussion über die Agenda 2010 hat dazu geführt, dass die Breite der Themen der Kanzlerrede vom 14. März 2003 verkürzt wiedergegeben wird. Die Agenda wurde auf den Bereich der Arbeitsmarktreformen, gelegentlich sogar auf Hartz IV reduziert ${ }^{13}$. Gerhard Schröder behandelte in seiner Rede neben der Außenpolitik mit den Schwerpunkten Irak und Europa drei Bereiche: „Konjunktur und Haushalt“, „Arbeit und Wirtschaft“ sowie „Reformen des Gesundheitswesens“. Hinzu kamen Forderungen zur Reform des Bildungssystems, die aber in der Fraktion unumstritten waren. Während sich die Aufmerksamkeit bereits im Vorfeld der Rede auf die Wirtschafts- und Arbeitspolitik konzentrierte, wurden die Haushaltsprobleme bei der Bewertung der Agenda unterschätzt. Sie standen aber in Schröders Konzept nicht ohne Grund nach der außenpolitischen Einleitung an erster Stelle.

\section{Konjunktur und Haushalt}

Der erste Hauptteil der Agenda-Rede galt den Haushalts- und Konjunkturfragen. Negative Wachstumsprognosen und sinkende Steuereinnahmen waren nach dem Wahlsieg der rot-

9 Vgl. GfV vom 3. Februar 2003 - 43.047. Die Fraktionsprotokolle der 14. und 15. Wahlperiode werden jeweils unter Angabe der Aktennummer im Archiv der sozialen Demokratie (AdsD), Friedrich-Ebert-Stiftung Bonn, wie folgt zitiert: $F=$ Fraktion, FV = Fraktionsvorstand, GfV = Geschäftsführender Fraktionsvorstand.

10 Vgl. F vom 10. Februar 2003 - 43.041; „Du solltest dich schämen!“, in: Der Spiegel vom 17. Februar 2003, S. 24.

11 Vgl. Karl-Rudolf Korte / Manuel Fröhlich, a.a.O. (Fn. 1), S. 297 f.; Matthias Geyer / Dirk Kurbjuweit / Cordt Schnibben, Operation Rot-Grün. Geschichte eines politischen Abenteuers, München 2005, S. $246 \mathrm{f}$.

12 Vgl. Daniel Friedrich Sturm, Wohin geht die SPD?, München 2009, S. 128.

13 Vgl. Anke Hassel / Christof Schiller, Der Fall Hartz IV. Wie es zur Agenda 2010 kam und wie es weitergeht, Frankfurt am Main 2010. 
grünen Koalition im September 2002 nicht mehr zu übersehen und wurden in der SPDFraktion seit dem Zusammentritt des 15. Bundestages ausführlich diskutiert. Die mit einem knappen Wahlergebnis bestätigte Regierung musste sich des Vorwurfs erwehren, sie habe die konjunkturelle Entwicklung im Wahlkampf zu optimistisch dargestellt und damit die Wähler getäuscht. Schröder wies diesen Vorwurf in der SPD-Fraktion zurück und verwies auf Konjunkturdaten, die sich seit September jede Woche geändert hätten. Der SPD müsse es bis zum Januar gelingen, die für sie negative und emotionale Stimmung zu „kippen“, um die am 2. Februar 2003 bevorstehenden Landtagswahlen in Niedersachsen und Hessen zu gewinnen ${ }^{14}$.

An der prekären Finanzlage änderten diese Beteuerungen und guten Vorsätze nichts: Nach der Steuerschätzung vom 13. November 2002 erklärte der neu gewählte Fraktionsvorsitzende Franz Müntefering im Fraktionsvorstand, die schwierige Finanzlage zwinge zu „Einsparungen in allen gesellschaftlichen Bereichen, die manches Wehgeschrei bei den Betroffenen hervorrufen würden"15. Finanzminister Hans Eichel und die Steuerfachleute der Fraktion kamen überein, dass für 2002 ein Nachtragshaushalt notwendig sei, der die Kreditaufnahme des Bundes auf 34,6 Milliarden Euro erhöhen würde. Im Folgejahr sollte die Kreditaufnahme wieder auf 18,9 Milliarden Euro und damit auf den Pfad der Tugend zurückgeführt werden. Am Fernziel, ab 2006 ohne Neuverschuldung auszukommen, wurde ausdrücklich festgehalten.

Dieses Fernziel sollte nach den Vorstellungen der rot-grünen Koalition durch die Streichung und Reduzierung von Steuerprivilegien und Subventionen erreicht werden. Die ersten Entwürfe des "Steuervergünstigungsabbaugesetzes" kamen, wie der Name vermuten lässt, aus dem Bundesfinanzministerium. Anfang Dezember 2002 legten die beiden Regierungsfraktionen gemeinsam einen Gesetzentwurf vor, der unter anderem die Begrenzung der Eigenheimförderung, die Mindestbesteuerung von Unternehmen, eine höhere Besteuerung von Dienstwagen und einen Verzicht auf Sonderregelungen bei der Ökosteuer vorsah. Die ermäßigte Mehrwertsteuer sollte nur noch für Güter und Dienstleistungen der „materiellen und kulturellen Grundversorgung" gelten. Von den circa 40 Einzelmaßnahmen des Gesetzes erhoffte sich Hans Eichel bereits im ersten Jahr Mehreinnahmen von mehr als 17 Milliarden Euro.

Das Steuervergünstigungsabbaugesetz stieß allerdings außerhalb des Regierungslagers auf erheblichen Widerstand. Steuerexperten und Wissenschaftler kritisierten vor allem die Mindestbesteuerung der Unternehmen. Die heftigste Kritik der Lobbyisten kam aus der Landwirtschaft: Der Weinbau wandte sich gegen die Besteuerung von Werbegeschenken. Die Gärtner protestierten gegen die erhöhte Mehrwertsteuer für Zierpflanzen und Schnittblumen. Sie erhielten Rückendeckung durch eine entsprechende Eingabe der Kolumbianischen Botschaft. Der Parlamentarische Staatssekretär im Ministerium für Verbraucherschutz, Ernährung und Landwirtschaft, Gerald Thalheim (SPD), unterstützte diese Forderungen in einem Schreiben an den Stellvertretenden Fraktionsvorsitzenden Michael Müller $^{16}$. Entscheidend für das Schicksal des geplanten Abbaus von Steuervergünstigungen und Subventionen war jedoch die Haltung des Bundesrats, in dem die CDU- und CSU-

14 Vgl. F vom 14. November $2002-46.085$.

15 FV vom 15. November $2002-43.046$.

16 Vgl. Schreiben Gerald Thalheim an Michael Müller (MdB) vom 13. Dezember 2002 und weitere Details in AdsD 47.807. 
geführten Regierungen die Mehrheit hatten. Franz Müntefering forderte vor der Fraktion, die SPD müsse die Union ,in die Pflicht“ nehmen. Finanzminister Eichel argumentierte in derselben Sitzung, die Haltung der Opposition werde zeigen, wer die Drei-Prozent-Grenze der Maastrichter Kriterien verletze. Regierung und SPD-Fraktion hofften dabei auf das Eigeninteresse der Länder, die von den Subventionskürzungen mit mehreren Milliarden profitiert hätten ${ }^{17}$.

Die CDU/CSU war aber nicht bereit, dieser Versuchung nachzugeben, und bewertete die Streichung von Steuerprivilegien als Steuererhöhungen. Ihre Haltung verhärtete sich nach den CDU-Wahlerfolgen in Niedersachsen und Hessen am 2. Februar 2003. Zwei Tage nach der Wahl forderte CDU-Generalsekretär Laurenz Meyer die Rücknahme des Steuervergünstigungsabbaugesetzes. Die CDU werde dem Paket nicht zustimmen. Gleichzeitig berichtete die Presse von der Bereitschaft des Bundeskanzlers, auf den Abbau von Steuervergünstigungen zu verzichten. Stattdessen sollten für 2003 eine höhere Neuverschuldung in Kauf genommen und die Maastricht-Kriterien erneut verletzt werden ${ }^{18}$.

Für die SPD-Fraktion kam diese Wende in der Finanzpolitik nicht überraschend, denn Schröder hatte bereits in der ersten Fraktionssitzung nach der Weihnachtspause die erhöhte Neuverschuldung im Nachtragshaushalt 2002 als „ein regelrechtes Konjunkturprogramm“ bezeichnet. Der Vorwurf, seine Regierung betreibe eine prozyklische Politik, sei angesichts der erneut reduzierten Wachstumsprognosen falsch. Notwendig sei vielmehr eine „aktivere Herangehensweise"19. Wenige Tage später erklärte Joachim Poß im Geschäftsführenden Vorstand, die Mitglieder des Haushaltsausschusses seien durch die negativen Prognosen zum Wirtschaftswachstum verunsichert. Nicht die Drei-Prozent-Grenze der Maastricht-Vereinbarung sollte das Thema sein, sondern die „Festigung der staatlichen Einnahmen“ 20 . Schröder schildert in seinen Erinnerungen selbstkritisch die „kommunikative Falle“, in der seine Regierung steckte: Auf der einen Seite standen das selbstverkündete Sparziel und die unflexiblen Maastricht-Kriterien, auf der anderen Seite drohte die Gefahr, als Konjunkturbremse zu wirken und die „Schwindsucht der Staatsfinanzen“ zu fördern ${ }^{21}$.

Unmittelbar vor und nach den Wahlniederlagen in Hessen und Niedersachsen wurden auch in der SPD-Fraktion kritische Stimmen zum „Steuervergünstigungsabbaugesetz “ laut: „Das Gesetz müsse nochmals auf seine ökonomischen Wirkungen untersucht werden“, notiert das Protokoll. Die Abgeordnete Brunhilde Irber kritisierte zum Beispiel die geplante Mehrwertsteuer für grenzüberschreitende Flüge, weil hierdurch deutsche Reiseunternehmen und Flughäfen benachteiligt würden. Ihr Fraktionskollege Jörg-Otto Spiller entgegnete, die Flugbesteuerung sei als Konzession an die Grünen aufgenommen worden und werde „am Ende so nicht im Gesetz stehen“22. Das Steuervergünstigungsabbaugesetz wurde jedoch zunächst weiter verfolgt. Bundeskanzler Schröder bat am 18. Februar 2003 die Fraktion, das Gesetz „in seiner derzeitigen Fassung“ im Bundestag zu beschließen, damit die

17 Vgl. F vom 14. Januar 2003 - 43.041.

18 Vgl. „Schröder erwägt Verzicht auf Steuerpaket“, in: Spiegel-Online vom 5. Februar 2003, http:// www.spiegel.de/politik/deutschland/0,1518,233781,00.html (Abruf am 14. Mai 2010); Susanne Höll / Robert Jacobi, Regierung erwägt Verzicht auf Steuerpaket, in: SZ vom 5. Februar 2003, S. 1.

$19 \mathrm{~F}$ vom 9./10. Januar $2003-43.041$.

20 GfV 13. Januar $2003-43.047$.

21 Vgl. Gerhard Schröder, Entscheidungen. Mein Leben in der Politik, Hamburg 2006, S. 436 438.

22 F vom 1./17./18. Februar $2003-43.041$. 
CDU/CSU gezwungen sei, „ihre Alternativen zu verdeutlichen“23. Nach Beratungen im Vermittlungsausschuss wurde schließlich mit Zustimmung der Opposition im April 2003 ein Steuervergünstigungsabbaugesetz beschlossen, das aber mit dem von Hans Eichel vorgelegten Entwurf nur noch wenig gemein hatte. Statt der zuletzt erwarteten 15 Milliarden rechnete man nur noch mit Mehreinnahmen von 4,4 Milliarden Euro ${ }^{24}$.

Gerhard Schröder musste bei seiner Agenda-Rede am 14. März 2003 vom Scheitern der geplanten Subventionskürzungen im Bundesrat ausgehen. Er erwartete deshalb vom Steuervergünstigungsabbaugesetz in Verbindung mit der geplanten Abgeltungssteuer im laufenden Jahr nur noch Einsparungen von einer Milliarde Euro. In Übereinstimmung mit den kritischen Stimmen in der Fraktion erklärte er, der Stabilitätspakt von Maastricht dürfe „nicht statisch interpretiert werden“. Während Schröder Ende Februar im Geschäftsführenden Vorstand der Fraktion ein Investitionsprogramm noch abgelehnt hatte, änderte er in den folgenden Tagen seine Meinung. Auf der Fraktionssitzung vom 11. März und in seiner Agenda-Rede versprach er Entlastungen für die Kommunen und ein Investitionsprogramm der Kreditanstalt für Wiederaufbau in Höhe von 15 Milliarden Euro. Gleichzeitig bestätigte er die bereits beschlossenen Steuerentlastungen ab Jahresbeginn 2004 und 200525.

Zur Abgeltungs- und Vermögenssteuer verliefen die Beratungen in der SPD-Fraktion weniger harmonisch. Die Abgeltungssteuer war Teil des Finanzpakets, das zusammen mit dem Steuervergünstigungsabbaugesetz von einer „Runde beim Bundeskanzler“ am 17. November 2002 in seinen Grundzügen vereinbart wurde. Ursprünglich sah man vor, Kapitalerträge und Spekulationsgewinne nur mit 15 Prozent zu versteuern. Dieser niedrige Satz veranlasste bereits in der nächsten Sitzung des Fraktionsvorstandes Jörg-Otto Spiller zu dem Einwand, damit schaffe man für die „Spekulanten“ Verbesserungen gegenüber dem geltenden Recht ${ }^{26}$. Die Vermögenssteuer wurde im Herbst 2002 von den Ministerpräsidenten der Länder Nordrhein-Westfalen und Niedersachsen, Peer Steinbrück und Sigmar Gabriel, gefordert. Der Fraktionsvorsitzende Müntefering erklärte hierzu im Geschäftsführenden Vorstand, wenn diese Länder hierfür im Bundesrat eine Mehrheit erreichten, „werde sich der Bund nicht sperren “27. Zwei Wochen später sagte er allerdings, „von der Abgeltungssteuer sei zu erhoffen, dass sie die Vermögenssteuerdebatte beendet". Joachim Poß hielt dem entgegen, die geplante Abgeltungssteuer sei kein Ersatz für die Vermögenssteuer. Sie sei allerdings eine „einmalige Chance“, die Steuerhinterziehung mittels Auslandskonten zu verhindern. Außerdem könne die Bundesregierung gegenüber der Öffentlichkeit eine "Verbesserung der Steuergerechtigkeit vorweisen“. Angelica Schwall-Düren stellte hierzu die kritische Frage, „warum ein Steuerhinterzieher durch eine Abgeltungssteuer von 25 Prozent einen steuerlichen Vorteil erlangen soll“. Michael Müller warnte angesichts der Diskussion davor, in der Öffentlichkeit den Eindruck zu erwecken, es bestünde ein Gegensatz zwischen den „steuerrechtlichen Positionen“ des Fraktionsvorsitzenden und des Bundeskanz-

23 F vom 18. Februar $2003-43.041$.

24 Vgl. „Eichels Sparplan auf ein Drittel zusammengestrichen“, in: Spiegel-Online vom 10. April 2003, http://www.spiegel.de/politik/deutschland/0,1518,244105,00.html (Abruf am 15. Mai 2010).

25 Vgl. F vom 11. März $2003-43.041$.

26 Vgl. FV vom 18. November 2002 - 43.046; Joachim Poß, Ziele der aktuellen finanzpolitischen Maßnahmen, 20. November $2002-43.040$.

27 GfV vom 2. Dezember $2002-43.047$. 
lers $^{28}$. Gerhard Schröder interpretierte in seiner Agenda-Rede den kontroversen Diskussionsstand als eindeutige Absage an die Vermögenssteuer. Sein Hauptargument war, die Abgeltungssteuer werde im Ausland angelegte Gelder nach Deutschland zurückholen. Die „Kehrseite“ dieser Steuer war nach seinen Worten, dass die „Substanz von Vermögen steuerfrei bleiben kann“, und er fügte bekräftigend hinzu: „Auch das muss klargestellt werden." 29

Im Bereich der Haushalts- und Finanzpolitik bestand demnach weitgehende Übereinstimmung zwischen Fraktion und Regierung. Die SPD-Abgeordneten waren am ursprünglichen Plan der Subventionskürzungen intensiv beteiligt und unterstützten zunächst den Sparkurs des Finanzministers. Als sich zeigte, dass der Abbau von Steuervergünstigungen im Bundesrat nicht durchzusetzen war und die Einwände der betroffenen Interessengruppen zahlreicher wurden, nahmen die kritischen Stimmen in der Fraktion zu. Mehrere Abgeordnete forderten wirksame Maßnahmen gegen die Arbeitslosigkeit und „Initiativen, die das Wachstum stimulieren" (Hans-Peter Bartels). Eichel lehnte noch Anfang Februar ein Investitionsprogramm „als Heilmittel“ ab. Der Kanzler bekräftigte diese Ablehnung zwei Wochen später vor dem Geschäftsführenden Fraktionsvorstand ${ }^{30}$. Unmittelbar vor seiner Rede am 14. März änderte er jedoch seine Meinung und kündigte in der Fraktion das bereits erwähnte Investitionsprogramm von 15 Milliarden Euro mit der Begründung an, „es gelte jetzt, Wachstumsimpulse zu setzen“" 31 . Dieser Positionswechsel ist als Konzession an den linken Flügel der Fraktion zu bewerten.

Die Frage, ob diese keynesianische Wende „richtig“ und der damaligen Wirtschaftslage angemessen war, sollte der Kontroverse unter den Ökonomen überlassen bleiben. Für die Fragestellung dieses Beitrags bleibt festzuhalten, dass die Aussagen der Agenda 2010 zur Haushalts- und Konjunkturpolitik keineswegs als einsame Entschlüsse des Kanzlers und seiner Entourage anzusehen sind. Sie ergaben sich vielmehr aus der Wirtschaftsentwicklung der vorangehenden Monate, aus den Mehrheitsverhältnissen im Bundesrat und aus den enttäuschenden SPD-Wahlergebnissen in Niedersachsen und Hessen. In diesem Bereich kann man deshalb durchaus von einer Mitregierung der Regierungsfraktion sprechen. Das bereits erwähnte Strategiepapier des Kanzleramts vom 5. Dezember 2002, oft als Vorläufer der Agenda 2010 interpretiert, bot wenig Hilfe für die finanzpolitische Situation zu Jahresbeginn 2003. Hier wurden noch „nachhaltig finanzierte“ Investitionen gefordert und eine Finanzierung über Verschuldung oder Steuererhöhungen ausgeschlossen ${ }^{32}$. In der Frage der Vermögenssteuer dagegen konnte Schröder seine ablehnende Haltung mit der in diesem Punkt unklaren Willensbildung in der Fraktionsführung und bei den Abgeordneten rechtfertigen. Die Ausführungen der Agenda zur Finanzpolitik verband er mit der Warnung vor einer Blockadepolitik des Bundesrates. Die Opposition könne auf diesem Gebiet zeigen, ob sie „Verantwortung für das Ganze“ übernehmen oder „,egoistisch ihr eigenes Süppchen kochen" wolle.

28 Vgl. GfV vom 16. Dezember 2002 - 43.047.

29 Regierungserklärung von Bundeskanzler Schröder am 14. März 2003 vor dem Deutschen Bundestag, hier und im Folgenden zitiert nach der Fassung von http://www.bundesregierung.de.

30 F vom 10. Februar 2003 - 43.041; GfV vom 24. Februar 2003 - 43.047.

31 F vom 11. März $2003-43.041$.

32 Vgl. FAZ, a.a.O. (Fn. 6). 


\section{Renten und Gesundheit}

Die Themen Rente und Gesundheit wurden in Schröders Rede am 14. März 2003 in unterschiedlichen Abschnitten behandelt. Sie standen aber in einem engen Zusammenhang, der sich aus der Vorgeschichte der Agenda ergab: Bereits zwei Wochen nach Abschluss der Koalitionsverhandlungen vom Oktober 2002 referierte die alte und neue Gesundheitsministerin Ulla Schmidt in der Fraktion über die notwendige Kostendämpfung bei der Krankenund Rentenversicherung und schlug eine Erhöhung des Rentenbeitrags von 19,1 auf 19,5 Prozent vor. Zu dieser Frage entwickelte sich ein handfester Konflikt zwischen den beiden Koalitionspartnern. Die Grünen bestanden nach dem Bericht Münteferings auf einer Beitragspflicht für Monatseinkommen bis zu 4.500 Euro und einem Beitragssatz von 19,3 Prozent, während die Sozialdemokraten bis zu einer Beitragsgrenze von 5.100 Euro einen Satz von 19,5 Prozent vorsahen ${ }^{33}$. Um den Konflikt, bei dem sich die sozialdemokratischen Vorstellungen durchsetzten, zu entschärfen, einigte sich die Gesundheitsministerin mit den Grünen, eine „Expertenkommission zur Reform der sozialen Sicherungssysteme“ einzusetzen - die spätere Rürup-Kommission. Bei der Abstimmung des Bundestages über das „Beitragssicherungsgesetz" am 15. November 2002 gaben 18 Abgeordnete der Grünen eine Erklärung zu Protokoll. Sie stimmten demnach dem Gesetz nur zu, weil die Einsetzung der Kommission die „Perspektive für eine grundlegende Reform“ "eröffne, bei der die Generationengerechtigkeit berücksichtigt und die Lohnnebenkosten gesenkt werden ${ }^{34}$.

Angesichts des Widerstands der Grünen bei der Rentenfinanzierung hatte sich inzwischen in der SPD-Fraktion eine Gegenaktion aus dem Gesundheitsbereich entwickelt: 22 Abgeordnete beantragten am Vorabend der Abstimmung über das Beitragssicherungsgesetz, die im Sparpaket vorgesehene fünfprozentige Kürzung der Leistungen für Zahntechniker rückgängig zu machen. Diese Maßnahme könne sich zu einer „negativen Symbolbedeutung für die Koordinationsfähigkeit sozialdemokratischer Politik entwickeln" (Christoph Zöpel). Das Zahntechnikerhandwerk habe seine 6.000 Mitglieder aufgefordert, SPD zu wählen (Horst Schmidbauer) und dürfe nicht enttäuscht werden. Ulla Schmidt dagegen verwies auf die angeblich über dem europäischen Durchschnitt liegenden deutschen Preise für Zahntechnik, und Franz Müntefering erklärte, die Grünen seien nicht bereit, die Änderung zu akzeptieren. Gegebenenfalls müsse das Gesetz von der Tagesordnung des Bundestages abgesetzt werden. Nach dem Hinweis, man könne „Ungerechtigkeiten dem Handwerk gegenüber" in der bevorstehenden Strukturreform des Gesundheitswesens ausgleichen (Helga Kühn-Mengel), wurde der Antrag der 22 bei wenigen Enthaltungen abgelehnt ${ }^{35}$.

Da sich Gesundheitspolitik und Rente als Konfliktbereiche in der Regierungskoalition und teilweise auch in der eigenen Fraktion erwiesen, reagierte Gerhard Schröder mit Vorsicht. Der Beschluss, die Rürup-Kommission einzusetzen, kam ihm dabei zur Hilfe. Anfang

33 Vgl. F vom 30. Oktober 2002 - 43.040; GfV vom 4. November 2002 - 43.047.

34 Da eine Recherche der Parlamentsprotokolle nach Datum im Online-Angebot des Deutschen Bundestages nicht mehr möglich ist, muss die Parlamentarismus-Forschung zunehmend auf Google zurückgreifen. Vgl. daher die Protokollerklärung zum Beitragssicherungsgesetz unter http://alex-bonde.de/themen/haushaltspolitik/generationen-gerechtigkeit/protokollerklaerungzum-beitragssicherungsgesetz-15112002.html (Abruf am 10. März 2011).

35 Vgl. F vom 14. November 2002 - 43.040 sowie Nils Bandelow / Anja Hartmann, Weder rot noch grün, in: Christiph Egle / Reimut Zohlnhöfer (Hrsg.), Ende des rot-grünen Projekts. Eine Bilanz der Regierung Schröder 2002-2005, Wiesbaden 2007, S. 334 - 354, S. 336 f. 
Januar 2003 verwies er in der Fraktion auf die zu erwartenden Eckpunkte aus dem Gesundheitsministerium sowie auf die Resultate der Rürup-Kommission. Beides dürfe nicht „auseinanderfallen“, wenn man die Gesundheitsreform „erfolgreich kommunizieren“ wolle. ${ }^{36}$ Unmittelbar vor seiner Agenda-Rede beschränkte sich Schröder in der Fraktion offenbar auf den Grundsatz, das medizinisch notwendige zur Wiederherstellung der Gesundheit solle nach wie vor solidarisch finanziert werden ${ }^{37}$. Am 14. März sprach er im Bundestag allerdings ausführlich über die Probleme des Gesundheitswesens, schilderte die Defizite des Systems und erklärte, die „Strategie der Kostendämpfung“ sei an ihre Grenzen gestoßen. Zwanzig Prozent der Kosten würden durch „Über- oder Fehlversorgung“ verursacht. Er nannte eine Reihe von Reformmaßnahmen, die man in der Fraktion vorher nicht diskutiert hatte: die Auflösung des Vertragsmonopols der Kassenärztlichen Vereinigungen, eine Verringerung der Zahl der Krankenkassen, die Reduzierung des Leistungskatalogs durch die Ausgliederung des Kranken- und Mutterschaftsgeldes, den elektronischen Patientenausweis etc. Das „kostenbewusste Verhalten“ von Patienten und Ärzten sollte durch Eigenbeteiligung, Selbstbehalte und „differenzierte Praxisgebühren“ gefördert werden. Schröder übernahm damit Forderungen aus dem Kanzleramtspapier vom 5. Dezember 2002, das aber mit dem Vorschlag von Wahltarifen und Beitragsrückerstattung noch darüber hinaus ging.

Die Forderungen des Kanzleramtspapiers zur Rentenfinanzierung waren deutlich und begannen mit der Feststellung, der Bund gebe fast jeden dritten Euro für die Rente aus. Aus Gründen der Gerechtigkeit müssten auch die Rentner an der „Rückführung der konsumtiven Ausgaben“ beteiligt werden. Hinzu kam ein wirtschaftspolitischer Gesichtspunkt: Die Rentenzahlungen, argumentierten die Autoren des Papiers, hätten eine hohe Sparquote und eine geringe Konsumquote zur Folge. Die Rürup-Kommission solle prüfen, wie man den „Ausgabenblock Rente“ in den nächsten Jahren verringern könne ${ }^{38}$. Schröder übernahm diese Forderungen nicht in seine Agenda-Rede. Er verwies auf die Anfangserfolge der Riester-Rente und sprach von der Notwendigkeit, „bei der Rentenversicherung nachzujustieren“. Von der Rürup-Kommission erwartete er Vorschläge für eine neue Rentenformel. Seine Zurückhaltung in diesem Punkt war auf Stimmen aus der Fraktion zurückzuführen. Die Parlamentarische Linke hatte bereits in ihrer Stellungnahme zum Kanzleramtspapier auf den hohen Anteil von Klein- und Kleinstrenten hingewiesen. Sie verband dieses Argument mit der grundsätzlichen Frage, ob man mit der Ankündigung von Kürzungen mehr Vertrauen und Zuversicht erreichen könne ${ }^{39}$.

Schröders Aussagen zur Gesundheitsreform dagegen kamen für die Fraktion überraschend. Hier ging man davon aus, dass sich die geplante Reform noch im Anfangsstadium befinde, denn Gesundheitsministerin Ulla Schmidt hatte erst Anfang 2003 erste Eckpunkte für einen Gesetzentwurf vorgelegt ${ }^{40}$. Das von Schröder angekündigte Reformplateau stieß aber keineswegs auf einhellige Ablehnung. Der linke Flügel der Fraktion äußerte sich kritisch, weil er die Wirkung der mit der Reform angestrebten Senkung der Lohnnebenkosten bezweifelte. Für die Netzwerker dagegen war die stärkere Eigenbeteiligung der Patienten „kein Tabu“. Der Seeheimer Kreis stellte sich in einem nach der Agenda-Rede verfassten

36 Vgl. F vom 9./10. Januar $2003-43.041$.

37 Vgl. F vom 11. März 2003 - 43.041.

38 Vgl. FAZ, a.a.O. (Fn. 6).

39 Vgl. Gernot Erler / Michael Müller / Angelica Schwall-Düren, a.a.O. (Fn. 8).

40 Vgl. Nils Bandelow / Anja Hartmann, a.a.O. (Fn. 35), S. 338 - 341. 
Papier noch deutlicher hinter die Forderungen Schröders. Er unterstützte die Aufhebung des Monopols der Kassenärztlichen Vereinigungen, die Reduzierung der Zahl der Krankenversicherungen und die Streichung des Krankengeldes aus dem Leistungskatalog. Was über das medizinisch Notwendige hinausgehe, hieß es, dürfe auf keinen Fall von der Solidargemeinschaft finanziert werden ${ }^{41}$.

Bei den Beratungen der SPD-Fraktion über Rente und Gesundheit ist eine zunehmende Distanz zum grünen Koalitionspartner nicht zu übersehen. Besonders deutlich wurde dies bei einer Sitzung des Fraktionsvorstandes Anfang Dezember 2002: Müntefering erklärte hier, die Grünen wollten die „sozialen Sicherungssysteme in ihrer heutigen Form im Wesentlichen aufgeben“. Die SPD solle sich deshalb nicht zu Stellungnahmen drängen lassen, bevor die Ergebnisse der Rürup-Kommission vorlägen. Olaf Scholz wies ebenfalls auf die „im Ansatz grundsätzlich unterschiedlichen Konzepte für die Weiterentwicklung der sozialen Sicherheitssysteme zwischen den Grünen und der SPD hin" 42 . Ob diese Unterschiede so grundlegend waren, mag angesichts der Zustimmung der Grünen zur Agenda 2010 dahingestellt bleiben. Doch auch bei den Beratungen zur Finanzpolitik warnte Joachim Po $\beta$ vor der „Öffentlichkeitswirkung“ der Grünen. Man müsse verhindern, dass diese sich „als Reformer gegenüber der SPD profilieren könnten" 43 . In der Fraktion blickte man nicht ohne Neid auf den kleineren Koalitionspartner, weil dessen Ergebnisse bei den Landtagswahlen stabil blieben.

Offenbar wollte Schröder mit seinen Vorschlägen zur Gesundheitspolitik auch die Positionen der SPD bei den bereits laufenden Gesprächen mit den Unionsparteien festlegen. In diesem Sinn ist sein Votum gegen die Ausgliederung des Zahnersatzes und der Sportunfälle aus der Krankenversicherung zu verstehen. Er verband es mit einem ausdrücklichen Appell an die Opposition, denn man werde in diesem Bereich nur weiterkommen, wenn die Mehrheiten in Bundestag und Bundesrat zu einer durchgreifenden Reform bereit seien. Müntefering hatte bereits am 10. Februar vor der Fraktion erklärt, die kommende Gesundheitsreform werde „ein sozialdemokratisches Gesetz sein, dass dann mit der CDU verhandelt werden kann“" ${ }^{4}$. Hier wird deutlich, dass Schröder die CDU/CSU nicht nur bei der Finanzpolitik, sondern auch bei der Gesundheitsreform zur Mitarbeit über den Bundesrat aufrief.

\section{Arbeit und Wirtschaft}

Der Kanzler bezeichnete seine Reformüberlegungen zum Bereich „Arbeit und Wirtschaft“ als das Herzstück seiner Reformagenda. Sie waren bereits bei der Formulierung der umstrittenste Teil seines Programms und sind es bis heute geblieben. Die Diskussionen hierzu in der SPD-Fraktion lassen sich bis zu den Beratungen der Kommission „Moderne Dienstleistungen am Arbeitsmarkt“ zurückverfolgen, die im August 2002 ihren Bericht vorlegte.

41 Vgl. „Reformen für Arbeit und Gerechtigkeit“, März 2003, S. 10 (dem Verfasser freundlicherweise von Ulrich Kelber, MdB, zur Verfügung gestellt); Mut zur Veränderung - Agenda 2010: Reformen für Deutschland, Seeheim Eckpunktepapier vom 29. April 2003, S. 3.

42 FV vom 2. Dezember $2002-43.046$.

43 GfV vom 16. Februar $2002-43.047$.

44 F vom 10. Februar $2003-43.041$. 
Die Empfehlungen dieses Gremiums, das nach seinem Vorsitzenden „Hartz-Kommission“ genannt wurde, waren zum Zeitpunkt der Agenda-Rede erst zum Teil von Bundestag und Bundesrat verabschiedet. Das Gesetz Hartz III, das in erster Linie die Reorganisation der Bundesanstalt für Arbeit betraf, und das Gesetz Hartz IV mit der Zusammenlegung von Arbeits- und Sozialhilfe befanden sich noch in der Planung. In der SPD-Bundestagsfraktion hatte man offenbar zunächst Vorbehalte gegen die Hartz-Kommission, denn die Parlamentarier waren in diesem Gremium nicht vertreten. Der damalige Fraktionsvorsitzende Peter Struck sah sich jedenfalls am 1. Juli 2002 zu der Feststellung veranlasst, nach der gemeinsamen Sitzung des SPD-Präsidiums und des Geschäftsführenden Fraktionsvorstandes „bleibe es bei der Linie, die Arbeit der Hartz-Kommission zu begrüßen“. Franz Thönnes fügte hinzu, auch im SPD-Parteirat habe es eine "gute Debatte" zur Hartz-Kommission gegeben ${ }^{45}$. Ihre Ergebnisse wurden der Öffentlichkeit am 16. August 2002 bei einem „Fachkongress“ im Französischen Dom am Berliner Gendarmenmarkt vorgestellt.

Eine Grundsatzdebatte in der Fraktion über diese Vorschläge wäre in den letzten Wochen vor der Bundestagswahl im September 2002 fehl am Platze gewesen. Stattdessen legten die beiden Regierungsfraktionen im Bundestag einen Unterstützungsantrag für die Hartz-Vorschläge vor ${ }^{46}$. Nach Bildung der zweiten rot-grünen Koalitionsregierung drangen der Bundeskanzler und der neue Wirtschafts- und Arbeitsminister Wolfgang Clement auf ihre „vollständige Umsetzung“. Die komplizierte Materie des Sozialrechts und der Blick auf den von der Opposition dominierten Bundesrat führten zu einer Aufteilung des Projekts in zustimmungsbedürftige und nicht zustimmungsbedürftige Gesetze. Den Bundestagsfraktionen der SPD und der Grünen blieb unter diesen Bedingungen wenig Zeit zur Beratung. Über politisch wichtige Details wurden sie oft mit Verspätung oder unzureichend informiert. Als die Ergebnisse des Vermittlungsausschusses zu Hartz I und II vorlagen, wurde in der SPD-Fraktion Kritik laut: Mehrere Redner befürchteten Einnahmeausfälle für die sozialen Sicherungssysteme durch die geringen Sozialbeiträge bei sogenannten Minijobs. Sie sahen außerdem die Gefahr, dass vor allem Frauen aus regulären Arbeitsverhältnissen in diese Minijobs gedrängt würden. Die Abgeordneten Ernst Dieter Rossmann, Angelica Schwall-Düren, Erika Lotz und Doris Barnett fragten nach den „Auswirkungen der HartzGesetzgebung auf die soziale Gerechtigkeit“ ${ }^{*}$. Kurz zuvor hatte die SPD-Fraktion der Hamburger Bürgerschaft versucht, eine Umfrage bei allen SPD-Landtagsfraktionen zu den voraussichtlichen finanziellen „Synergieeffekten“ der geplanten Zusammenlegung von Arbeits- und Sozialhilfe zu starten. Diese Aktion wurde jedoch von der Geschäftsstelle der Berliner Fraktionsführung gestoppt ${ }^{48}$.

Der Kontakt des Bundeskanzlers und seiner wichtigsten Minister, Wolfgang Clement und Hans Eichel, zu ihrer Fraktion war in dieser Situation enger als bisher angenommen. Das bereits erwähnte Thesenpapier des Bundeskanzleramts „Auf dem Weg zu mehr Wachstum, Beschäftigung und Gerechtigkeit" vom Dezember 2002 war bereits vor seiner Veröffentlichung in der Fraktion bekannt. Am 20. Dezember fand hierüber das bereits erwähnte Gespräch Schröders mit den Stellvertretenden Fraktionsvorsitzenden Angelica Schwall-Düren,

45 Vgl. FV vom 1. Juli $2002-42.901$.

46 Vgl. BT-Drs. 14/9946 vom 11. September 2002.

47 FV vom 16. Dezember $2002-43.046$.

48 Rundschreiben der Bund-Länder-Koordinierungsstelle der SPD-Bundestagsfraktion vom 12. Dezember 2002 (AdsD 48.140). 
Gernot Erler und Michael Müller statt, die als Vertreter der Parlamentarischen Linken anzusehen sind. In ihrer Stellungnahme zum Kanzleramtspapier und zu den Ausführungen Schröders erklärten die drei Abgeordneten, wenn die neuen Gesetze zum Arbeitsmarkt das „Fordern“ betonen, sei es umso wichtiger zu zeigen, wie auf diesem Wege mehr Gerechtigkeit und die Erneuerung des Sozialstaates erreicht werden könne. Zur Kritik im Kanzleramtspapier an den zu hohen Lohnnebenkosten verwiesen sie auf die hohe Produktivität, welche die starke Stellung Deutschlands auf den Exportmärkten ermögliche. Dass der Schlüssel für Wachstum und Beschäftigung in erster Linie bei den Investitionen liege, sei ein „konservativer Irrglaube“. Unter Berufung auf Ludwig Erhard entgegneten sie, ein dauerhaftes Wachstum benötige sowohl Investitionen als auch steigende Masseneinkommen ${ }^{49}$.

Auf der Klausurtagung der SPD-Fraktion am 9. und 10. Januar 2003 wurden neben den Haushaltproblemen die Arbeitsmarktreformen diskutiert. Clement stellte die Verbindung zwischen beiden Bereichen her, als er die Hoffnung aussprach, mit Hilfe des Hartz-Konzepts werde es gelingen ,auch bei verminderten Wachstumsprognosen politische Entwicklungen am Arbeitsmarkt anzustoßen". Bei den Beratungen über Lohnnebenkosten und Sozialstaat werde es nicht reichen, „das Vorhandene zu verteidigen“. Die Sozialsysteme müssten „effizienter“ werden, und jeder müsse mehr Eigenverantwortung tragen. Schröder bekräftigte auf dieser Tagung seine Auffassung, es bestehe eine enge Beziehung zwischen der Höhe der Lohnnebenkosten und der Höhe der Arbeitslosigkeit ${ }^{50}$.

Nach den Landtagswahlen in Hessen und Niedersachsen am 2. Februar 2003 musste der Fraktionsvorsitzende Müntefering einräumen, dass die Niederlagen der SPD einen „deutlichen bundespolitischen Anteil hatten“. Dieser erneute Rückschlag führte dazu, dass die kritischen Stimmen in der Fraktion zunahmen. Sie galten in erster Linie den Reformen am Arbeitsmarkt und weniger den Finanzproblemen, die man als unabwendbares Ergebnis der Wirtschaftsentwicklung bewertete. Schröder konzentrierte sich bei seiner Rede vor der Fraktion am 10. Februar auf die Außenpolitik und den drohenden Krieg im Irak. Dies veranlasste am folgenden Tag mehrere Fraktionsmitglieder zu der Kritik, die Wahlniederlagen seien kaum zur Sprache gekommen. Auf der Sitzung kritisierten zehn Redner die „mangelnde Beteiligung der Fraktion an Reformprojekten“. Andere befürchteten, die SPD verliere ihre „soziale Kompetenz“. Mehrere Abgeordnete forderten kurzfristig wirksame Schritte zum Abbau der Arbeitslosigkeit. Klaus Brandner, Joachim Poß und Wolfgang Clement wiesen dagegen auf die bisherigen Erfolge der Reformen hin. Franz Müntefering sagte zum Schluss der kontroversen Diskussion, nach 50 Jahren Wachstum und Wohlstand müsse man nun die „soziale Gerechtigkeit auf niedrigerem Niveau organisieren “51.

Eine Woche später wurde die Kritik an der Arbeitsmarktpolitik noch deutlicher. Mehrere Mitglieder des Fraktionsvorstands beklagten Kürzungen von Arbeitsbeschaffungsmaßnahmen (ABM). Rainer Wend bezeichnete die Parteiveranstaltungen vor Ort als „schrecklich“. Im Osten hätten die Hartz-Gesetze überhaupt keinen positiven Effekt. "Hartz" helfe nur bei Wachstum, nicht aber, Wachstum zu fördern. Hans-Peter Kemper erklärte, in der Öffentlichkeit entstehe der Eindruck, dass man nicht die Arbeitslosigkeit, sondern die Arbeitslosen bekämpfe. „Hartz“ sei richtig, wirke jedoch nicht in ökonomisch

49 Vgl. Gernot Erler / Michael Müller / Angelica Schwall-Düren, a.a.O. (Fn. 8).

$50 \mathrm{Vgl}$. F vom 9./10. Januar 2003 - 43.041.

51 F vom 11. Februar und 10. März 2003 - 43.041. 
schwachen Zeiten. Müntefering sprach jetzt von einer „gewissen Dynamik, die man nicht einfach laufen lassen könne" 52 . Der Fraktionsvorsitzende hatte schon auf der Fraktionsversammlung am 10. Februar erklärt, bei der vorangehenden Sitzung des SPD-Parteivorstands habe das Thema Sonderparteitag keine Rolle gespielt. Daraus lässt sich ableiten, dass ein solcher Parteitag zu diesem Zeitpunkt im Gespräch war. In der Fraktion plädierten Willi Brase und Ernst Dieter Rossmann für mehrere Regionalkonferenzen an Stelle eines Sonderparteitags ${ }^{53}$.

Am 24. Februar trug Gerhard Schröder im kleinen Kreis des Geschäftsführenden Fraktionsvorstands seine Überlegungen für die am 14. März geplante Regierungserklärung vor und stand den Abgeordneten zweieinhalb Stunden Rede und Antwort. Wie weit er sich dabei auf die Details der Arbeitsmarkt- und Sozialpolitik einließ, ist aus dem schriftlichen Protokoll kaum zu entnehmen. Diskutiert wurden bei dieser Gelegenheit neben dem IrakKonflikt die Lockerung des Kündigungsschutzes, der Abbau der ABM-Maßnahmen und die Finanzprobleme der Kommunen. Nachdem Eichel über die Haushaltsprobleme referiert hatte, berichtete und diskutierte Clement nahezu zwei Stunden lang mit den Vorstandsmitgliedern die Fragen der Wirtschafts- und Arbeitsmarktpolitik, wobei die Hartz-Gesetze im Mittelpunkt standen ${ }^{54}$.

In den Wochen vor der Agenda-Rede sorgten drei Themen in der Fraktion für Verstimmung: Die geplanten Regeln zur Zeitarbeit, zum Kündigungsschutz und zum Arbeitslosengeld stießen vor allem beim Gewerkschaftsflügel auf Widerstand, der auch über die Verkündung der Agenda hinaus bestand. Im November 2002 kam es in der SPD-Fraktion zunächst zu einem Disput über die Löhne der Zeit- und Leiharbeiter: Die Hartz-Kommission und Gerhard Schröder gingen davon aus, dass die Bezahlung der von den PersonalService-Agenturen an die Betriebe ausgeliehenen Arbeitnehmer deutlich unter den Löhnen der Stammbelegschaft liege. In der Sitzung des Fraktionsvorstandes am 4. November 2002 erklärte jedoch Klaus Brandner, der der IG-Metall nahe stand, es gelte das Prinzip „gleiche Bezahlung für gleiche Arbeit“. Die Zeitarbeiter müssten nach einer sechswöchigen Probezeit gleich bezahlt werden wie die anderen Arbeitnehmer im Betrieb ${ }^{55}$. Unmittelbar vor der Abstimmung über die beiden ersten Hartz-Gesetze erläuterte Wolfgang Clement die ins Gesetz aufgenommene Möglichkeit, hiervon durch gesonderte Tarifverträge abzuweichen. Gewerkschaften und Verleihfirmen hätten sich bereits auf eine Verlängerung der seinerzeit geltenden Tarifverträge bis 2004 geeinigt $^{56}$. Eine gleiche Bezahlung der Zeitarbeiter war demnach nicht geplant. Diese Regelung im Hartz I-Gesetz entsprach dem Vorschlag der Kommission, dem auch die Gewerkschaftsvertreter zugestimmt hatten ${ }^{57}$.

Die zweite umstrittene Frage betraf den Kündigungsschutz in Kleinbetrieben. Die Hartz-Kommission beabsichtigte, den bestehenden Kündigungsschutz nicht zu verändern und die Flexibilität auf dem Arbeitsmarkt durch eine großzügige Regelung der Zeit- und Leiharbeit zu erreichen. Weder im Bericht der Kommission noch in den Koalitionsverein-

52 FV vom 17. Februar $2003-43.046$.

53 Vgl. F vom 10. Februar $2002-43.041$.

54 Vgl. GfV vom 24. Februar $2003-43.047$

55 Vgl. FV vom 4. November $2002-43.046$

56 Vgl. F vom 14. November $2002-43.040$.

57 Vgl. hierzu das Interview mit Peter Hartz, „Mein Part ist erfüllt“, in: Der Spiegel vom 25. November 2002, S. $31-33$. 
barungen oder im Papier des Bundeskanzleramts vom 5. Dezember 2002 wurde eine Änderung des Kündigungsschutzes erwähnt. Zur Diskussion gestellt wurde dieses Thema durch den neuen Wirtschafts- und Arbeitsminister Clement, der sich bereits vor seiner Ernennung für eine Lockerung des Kündigungsschutzes in Betrieben mit mehr als fünf Beschäftigen ausgesprochen hatte. Er stützte sich hierbei auf ein Positionspapier seines Amtsvorgängers Werner Müller, das die Ergebnisse der Hartz-Kommission durch „weitergehende Vorschläge" ergänzte ${ }^{58}$. Clement wiederholte seine Forderung Mitte Januar 2003 mit dem Argument, wenn in Betrieben ab sechs Beschäftigten der Kündigungsschutz gelte, sei man wenig geneigt, mehr als fünf Mitarbeiter einzustellen. Er stieß diesmal auf heftige Proteste der Gewerkschaften, die darauf hinwiesen, dass die rot-grüne Koalition erst 1998 die Schwelle für den Kündigungsschutz von zehn auf fünf Mitarbeiter gesenkt hatte ${ }^{59}$.

In der SPD-Bundestagsfraktion wurde die Diskussion über den Kündigungsschutz nach den Wahlniederlagen in Niedersachsen und Hessen als ,wenig hilfreich“ bezeichnet ${ }^{60}$. Als der Bundeskanzler Ende Februar 2003 vor dem Geschäftsführenden Fraktionsvorstand seine geplante Regierungserklärung erläuterte, fragte Ludwig Stiegler nach der Lockerung des Kündigungsschutzes. Schröder ging offenbar auf dieses Thema nicht näher ein und verwies „auf die schon getroffenen Maßnahmen zur Flexibilisierung des Arbeitsmarktes"61. Erst unmittelbar vor der Agenda-Rede kam es zu einer Aussprache über diese wichtige Frage. Im Anschluss an Schröders Bericht über seine geplante Regierungserklärung votierten Ludwig Stiegler, Ottmar Schreiner, Erika Lotz und Wolfgang Grotthaus gegen die Lockerung des Kündigungsschutzes. Klaas Hübner und Volker Kröning entgegneten, man müsse auch den Mut haben, diese Frage „ohne ideologische Festlegungen“ zu diskutieren. Der Fraktionsvorsitzende Müntefering war offenbar von dieser Kontroverse überrascht und erklärte, es sei sicher notwendig „einmal offen“ über den Kündigungsschutz zu sprechen. Er selbst wolle den Kündigungsschutz nicht opfern, werde aber der Mehrheit folgen ${ }^{62}$. In seiner Rede am 14. März schlug Schröder dann nicht nur eine Reform des Kündigungsschutzes für kleine Betriebe, sondern auch Abfindungen bei betriebsbedingten Kündigungen und eine Änderung der so genannten Sozialauswahl des zu kündigenden Personals vor. Diese Vorstellungen zur Revision des Kündigungsschutzes gingen über die Hartz-Vorschläge hinaus. Sie wurden der Fraktion in letzter Minute eröffnet, obwohl es sich für die Gewerkschafter um Prinzipienfragen handelte.

Ein drittes Thema, mit dem die Bundestagsfraktion überrascht wurde, war die Bezugsdauer des Arbeitslosengeldes - seit den Hartz-Vorschlägen „Arbeitslosengeld I“ genannt. Bereits im März 2002 schlug der designierte Leiter der Bundesanstalt für Arbeit, Florian Gerster (SPD), vor, die Zahlung des Arbeitslosengeldes von maximal 32 Monaten stufenweise zu kürzen. Er stieß damit auf energische Proteste aus der SPD-Bundestagsfraktion, unter anderem von Gerd Andres, Franz Thönnes und Ottmar Schreiner ${ }^{63}$. In der Hartz-Kom-

58 Vgl. Hendrik Munsberg, Lockert Clement den Kündigungsschutz?, in: Berliner Zeitung vom 10. Oktober 2002.

59 Vgl. Patrick Welter, Clement legt sich mit Gewerkschaften an, in: FAZ.net vom 17. Januar 2003 (Abruf am 14. August 2010).

60 So Walter Hoffmann, F vom 10. Februar 2003 - 43.041.

$61 \mathrm{GfV}$ vom 24. Februar 2003 - 43.047.

62 Vgl. F vom 11. März 2003 - 43.041.

63 Vgl. „Gersters grummelige Genossen“, in: Spiegel-Online vom 4. März 2002, http://www.spiegel.de/wirtschaft/0,1518,185410,00.html (Abruf am 6. Juni 2010). 
mission verzichtete man in Übereinstimmung mit den Gewerkschaftsvertretern auf eine Kürzung des Arbeitslosengeldes und konzentrierte sich auf die Deregulierung der Zeitarbeit. Das Arbeitslosengeld I wird im Bericht der Kommission als „beitragsfinanzierte originäre Versicherungsleistung“ bezeichnet, die „in Höhe und Dauer im Grundsatz dem bisherigen Regelwerk" entsprechen soll ${ }^{64}$. Gerster meldete sich nach der Vorstellung der Hartz-Vorschläge erneut zu Wort und kritisierte, dass die Experten nicht den Mut gehabt hätten, das Arbeitslosengeld auf zwölf Monate zu begrenzen. Auf der SPD-Parteikonferenz am 18. August 2002 forderte daraufhin ein Delegierter, gegen Gerster ein Parteiordnungsverfahren einzuleiten ${ }^{65}$. Da das Arbeitslosengeld I weder im Koalitionsvertrag noch im Papier des Bundeskanzleramts erwähnt wurde, sah die SPD-Fraktion keine Veranlassung, sich mit diesem Thema zu befassen. Auch als Schröder den Geschäftsführenden Fraktionsvorstand über seine geplante Agenda-Rede informierte, wurde das Arbeitslosengeld I weder in seinem Bericht noch in der Diskussion erwähnt. Das gleiche gilt für die nahezu zweistündige Diskussion mit Wolfgang Clement in derselben Sitzung ${ }^{66}$. Die Forderung nach Kürzung des Arbeitslosengeldes I auf zwölf Monate in der Agenda 2010 traf die Fraktion daher unvorbereitet.

Die Zusammenlegung von Arbeitslosen- und Sozialhilfe kam in der Fraktion nur beiläufig zur Sprache, weil für das entsprechende Gesetz Hartz IV noch ausführliche Verhandlungen mit den Ländern und dem Bundesrat erforderlich waren. Dabei blieb unbeachtet, dass die Hartz-Kommission zur Höhe des neuen Arbeitslosengeldes II keine präzisen Angaben gemacht hatte. Während das „Sozialgeld“ für Nicht-Arbeitsfähige der bisherigen Sozialhilfe entsprechen sollte, beschreibt der Bericht die Leistung für die arbeitsfähigen Arbeitslosen nur in allgemeinen Wendungen: Sie sei „bedürftigkeitsabhängig“ und müsse den „Finanzstatus“ sowie den „familiären Kontext“ der Arbeitslosen berücksichtigen. Gleichzeitig hatte sich die Hartz-Kommission nach kontroverser Diskussion gegen kollektive Leistungskürzungen entschieden ${ }^{67}$. Die Parlamentarier konnten deshalb davon ausgehen, dass bei der Arbeitslosenhilfe alles „beim Alten“ bleiben würde.

$\mathrm{Zu}$ einer ersten Kontroverse über diese Frage kam es, als der Parlamentarische Staatssekretär im Wirtschafts- und Arbeitsministerium, Gerd Andres, dem Fraktionsvorstand am 4. November 2002 die Einsparmöglichkeiten bei der Arbeitslosenhilfe durch die Anrechnung von Einkommen und Vermögen des Lebenspartners erläuterte. In der darauf folgenden Fraktionssitzung wurde kritisiert, mit dieser Anrechnung sei die „Gefahr des Absinkens in die Armut“ verbunden. Die Regelung sei „auch im Hinblick auf die geplante Senkung des Spitzensteuersatzes auf 42 Prozent nicht vertretbar“", denn diese koste mehr Geld, als durch das Arbeitslosengeld II gespart werde ${ }^{68}$. Anschließend verlor die Fraktion dieses Thema offenbar aus dem Blickfeld. In der Fraktionsversammlung am 11. Februar 2003, an der auch Wolfgang Clement teilnahm, wurde darauf hingewiesen, dass die Zusammenlegung der Arbeits- und Sozialhilfe Ende März auf die Tagesordnung gesetzt werde. Auf der Sitzung des

64 Vgl. Hartz-Kommission, Moderne Dienstleistungen am Arbeitsmarkt, Berlin 2002, S. 125; Peter Hartz, a.a.O. (Fn. 57).

65 Vgl. Thomas Wittke, Nicht zerreden, zerschreiben, zersenden, in: General-Anzeiger Bonn vom 19. August 2002.

66 Vgl. GfV vom 24. Februar 2003 - 43.047.

67 Vgl. Anne-Marie Weimar, Die Arbeit und die Entscheidungsprozesse der Hartz-Kommission, Wiesbaden 2004, S. 105, S. 135.

68 Vgl. FV vom 4. November 2002 - 43.046; F vom 5. November 2002 - 43.040. 
Geschäftsführenden Fraktionsvorstandes am 24. Februar kam die Zusammenlegung weder in den ausführlichen Referaten Schröders und Clements noch in der Diskussion zur Sprache. Entsprechendes gilt für die Fraktionssitzung am 11. März, auf der der Kanzler über die Vorbereitung der Agenda-Rede berichtete ${ }^{69}$. Selbst in den Stellungnahmen der Parlamentarischen Linken und der Netzwerker wurde die später so brisante Frage nach der Höhe des Arbeitslosengeldes II nicht erwähnt ${ }^{70}$. Die Erklärung Schröders in seiner Agenda-Rede, das neue Arbeitslosengeld solle „in der Regel dem Niveau der Sozialhilfe entsprechen“, kam deshalb für die Fraktion überraschend.

\section{Bilanz: teils mitsteuernde, teils überraschte Fraktion}

Die Agenda 2010 ist eine Variante der von Gerhard Schröder seit Beginn seiner Kanzlerschaft gestarteten Reformversuche. Seine (nicht gehaltene) Rede zu Anthony Giddens' „Der dritte Weg" vom 11. März 1999, das Schröder-Blair-Papier vom Juni desselben Jahres, sein Aufsatz über die „zivile Bürgergesellschaft“ (April 2000) und die Berliner Konferenz „Modernes Regieren" vom Juni 2000 waren programmatische Initiativen, die auf die Reformbereitschaft der SPD zielten ${ }^{71}$. Mit dem Bündnis für Arbeit verfolgte Schröder gleichzeitig das Ziel, Reformen im Konsens der gesellschaftlichen Gruppen zu erreichen. Dieser Versuch scheiterte allerdings, bevor er im März 2003 offiziell aufgegeben wurde. Auch die HartzKommission bemühte sich, sowohl den Gewerkschaften als auch den Wirtschaftsverbänden gerecht zu werden. Die als Agenda 2010 bezeichnete Regierungserklärung war dagegen eine gouvernementale Aktion und wurde in der Erinnerung Gerhard Schröders „von einem sehr kleinen Kreis konzipiert". Nach den bisher vorliegenden Untersuchungen, die sich überwiegend auf die Presse stützen, war hierbei die Staatssekretärsebene bedeutender als die Minister.

Wie die Durchsicht der Fraktionsprotokolle zeigt, wurden die Themen der Agenda-Rede in der SPD-Fraktion seit der erneuten Bildung der rot-grünen Koalition im Oktober 2002 ausführlich diskutiert. Die Fragen des Arbeitsmarktes waren in der Fraktion stets präsent, weil unmittelbar nach der Regierungsbildung die ersten beiden Hartz-Gesetze in Angriff genommen wurden. Auch die Kosten des Gesundheitswesens kamen zur Sprache: Die Einsetzung der Rürup-Kommission löste offenbar wenig Begeisterung aus, denn der Fraktionsvorsitzende Franz Müntefering appellierte an die Abgeordneten, man möge die Kommission „in nicht-öffentlichem Dialog" begleiten ${ }^{72}$. Die Regierungsmitglieder beteiligten sich an dieser Diskussion in der Fraktion: Gerhard Schröder sprach zum Beispiel am 14. November 2002 vor der Fraktion zur Wirtschafts- und Finanzpolitik. Die Wirtschaftslage und die Haushaltssituation bildeten auch die Hauptthemen der Klausurtagung der Fraktion am 9. und 10. Januar 2003, auf der Schröder sowie der Wirtschafts- und Arbeitsminister Wolfgang

69 Vgl. F vom 11. Februar 2003 - 43.041; GfV vom 24. Februar 2003 - 43.047; F vom 11. März $2003-43.041$.

70 Vgl. Gernot Erler / Michael Müller / Angelica Schwall-Düren, a.a.O. (Fn. 8); „Reformen für Arbeit und Gerechtigkeit“, a.a.O. (Fn. 41).

71 Vgl. Sebastian Fischer, Gerhard Schröder und die SPD, München 2005, S. 71 - 80, S. 92 - 96; Karlheinz Niclauß, Kanzlerdemokratie, a.a.O. (Fn. 1), S. $346-349$.

72 Vgl. F vom 10. Januar $2003-43.041$. 
Clement referierten. Zwischen der Bundestagswahl vom 22. September und der AgendaRede nahm Schröder an elf, Clement an zwölf Fraktionssitzungen teil ${ }^{73}$.

Die entscheidende Frage ist, was bei diesen Gesprächen vermittelt wurde. Konnten die Fraktionsmitglieder bereits in der Vorbereitungsphase der Agenda im Sinne einer „Mitsteuerung " tätig werden? Hat die SPD-Fraktion die mit der Agenda verbundene Richtungsentscheidung kontrolliert und ihre Bedeutung für kommende Wahlen sowie für die sozialdemokratische „Basis“ in ihre Überlegungen einbezogen? Wenn man die Themen der Agenda 2010 unter diesem Gesichtspunkt betrachtet, nehmen Schröders Ausführungen zu „Konjunktur und Haushalt" vor der Fraktion eine Sonderstellung ein. Dieser erste Hauptteil seiner Rede entstand in engem Kontakt mit der Bundestagsfraktion. Er unterscheidet sich auch deutlich von den Vorschlägen des Kanzleramtspapiers vom 5. Dezember 2002. Die Zusage eines Konjunkturprogramms kann man als Konzession an den linken Flügel der Fraktion interpretieren, während zum Beispiel die Netzwerker noch Anfang März 2003 „kreditfinanzierte Konjunkturprogramme“ ausdrücklich ablehnten ${ }^{74}$.

Die beiden anderen Hauptteile der Rede, „Arbeit und Wirtschaft“ sowie Schröders Vorschläge zur Gesundheitsreform, wurden sehr diskret vorbereitet. Die einschneidenden Reformschritte blieben offenbar unerwähnt und führten deshalb in der SPD-Fraktion auch nicht zu Kontroversen. In den Berichten des Bundeskanzlers und des Wirtschaftsministers Clement ging es nur um die großen Linien der Regierungserklärung am 14. März 2003. Bereits bei der Vorstellung der Ergebnisse der Hartz-Kommission gab Schröder die Parole aus: „Nichts zerreden, zerschreiben, zersenden.“75 Laut Bericht des „Spiegels“ begründete SPD-Generalsekretär Olaf Scholz diese Kabinettspolitik bei der Vorbereitung der Agenda mit der Gefahr vorschneller Presseveröffentlichungen. Schröder rede bei seinen Gesprächen mit der Fraktion und den SPD-Bezirksvorsitzenden noch nicht „Klartext“, weil am nächsten Tag alles in der Zeitung stehe und die „Wirkung seines Masterplans (...) verpufft" ${ }^{\text {“76. }}$ Schröder selbst schreibt, von der „explosiven Kraft“ der Agenda habe im Vorfeld kaum jemand Notiz genommen, weil der drohende Irakkrieg zu dieser Zeit alle anderen Themen überschattete: „So konnten wir geräuschlos weiterarbeiten.“ In der Öffentlichkeit, in der Fraktion und in den Parteigremien habe er den „politischen Hintergrund“ der Agenda 2010 hinreichend deutlich gemacht. Details der Rede vom 14. März habe er jedoch erst am Vortag eingefügt, „um die Opposition zu überraschen“77.

Die in den strittigen Punkten geheime Vorbereitung der Agenda 2010 überraschte aber auch die eigene Fraktion. Während man auf dem Gebiet der Finanzpolitik von einer „Mitsteuerung“ der Bundestagsfraktion bei der Vorbereitung der Agenda sprechen kann, war dies in den Bereichen Gesundheits-, Arbeits- und Sozialpolitik nicht der Fall. Vor allem die über den Bericht der Hartz-Kommission hinausgehenden Maßnahmen mussten in der Fraktion Verunsicherung und Widerstand hervorrufen. Gerhard Schröder setzte hier auf das Verfahren der „brinkmanship“, das er bereits in der ersten Amtsperiode der rot-grünen

73 Vgl. Rednerlisten in 43.045, Archiv der sozialen Demokratie Bonn, SPD-Bundestagsfraktion, 15. Wahlperiode.

74 Vgl. „Reformen für Arbeit und Gerechtigkeit“, a.a.O. (Fn. 41), S. 5.

75 Thomas Wittke, a.a.O. (Fn. 65).

76 Dirk Kurbjuweit / Roland Nelles / Gabor Steingart, Reden um zu überleben, in: Der Spiegel vom 10. März 2003, S. 22 - 26, S. 24.

77 Gerhard Schröder, Entscheidungen. Mein Leben in der Politik, S. 391 ff. 
Koalition bei außenpolitischen Entscheidungen praktiziert hatte ${ }^{78}$. Die Zustimmung aus Überzeugung wurde auf diese Weise ergänzt durch die Zustimmung aus Furcht vor dem Kanzlerrücktritt und dem Scheitern der Regierungskoalition. Man gewinnt den Eindruck, Schröder habe seine harte Haltung gegenüber einigen Interessengruppen nach dem Scheitern des Bündnisses für Arbeit auf die eigene Fraktion übertragen. Jedenfalls bestand ein deutlich sichtbares Defizit an Kommunikation, das nicht nur bei der Vorbereitung, sondern auch im Text der Agenda selbst zum Ausdruck kam. Eine Begründung der Leistungskürzungen und eine damit verbundene Zukunftsperspektive fehlten. Da der „politische Diskurs" in der Sozialdemokratie in traditionellen Bahnen verlief, war innerhalb der Partei die Stimmung vorherrschend, man betreibe eine „Reformpolitik mit schlechtem Gewissen"79.

Obwohl die Reformvorschläge „von oben“ kamen, war die Unterstützung für die Agenda in der Fraktion breiter, als man unter dem Eindruck der späteren Auseinandersetzungen annimmt. Soweit sich aus den Fraktionsprotokollen entnehmen lässt, wurden Schröders Forderungen vom einflussreichen Seeheimer Kreis und der jungen Abgeordnetengruppe Netzwerk unterstützt. Die Vorbehalte der Linken waren deutlich erkennbar, aber nur bei einer kleinen Minderheit so stark, dass ein abweichendes Abstimmungsverhalten drohte. Während im August 2001 noch 18 SPD-Abgeordnete gegen den Mazedonien-Einsatz der Bundeswehr votierten, stimmten im September 2003 nur sechs Abgeordnete gegen die mit dem Bundesrat ausgehandelte Gesundheitsreform. Die Spannungen in der Fraktion wurden allerdings durch Schröders Forderung nach einer „eigenen Mehrheit“ der Regierungsparteien bei den Abstimmungen im Bundestag verschärft. Eine „eigene Mehrheit“ kennt das Grundgesetz nicht. Sie zu verlangen scheint wenig sinnvoll, wenn es sich um Gewissensentscheidungen oder um Gesetze handelt, die als Kompromiss mit der Opposition zustande kommen. Der Protest einer Fraktionsminderheit kam auch in dem Versuch zum Ausdruck, eine Abstimmung der SPD-Parteimitglieder über die Agenda durchzusetzen. Die Initiative hierzu ging von zwölf Bundestagsabgeordneten aus und wurde ebenso diskret vorbereitet wie die Agenda selbst. Sie blieb aber ohne Erfolg, weil die Zahl der erforderlichen Unterschriften (zehn Prozent der Parteimitglieder, das heißt circa 70.000 Unterschriften) bei weitem nicht erreicht wurde. Gerhard Schröder und die Parteiführung mussten jedoch einen Sonderparteitag zulassen, der am 1. Juli 2003 die Agenda 2010 mit deutlicher Mehrheit billigte. Hier und auf dem regulären Bochumer SPD-Parteitag im November konnte Schröder die nach wie vor kontrovers diskutierten Sachfragen „in Machtfragen umwandeln" 80 .

Das Verhältnis von Fraktion und Reformpolitik ist allerdings nur ein Ausschnitt aus dem Gesamtbild der Regierungszeit Schröders. Durch das Studium der Fraktionsprotokolle werden einige Fragen beantwortet. Weitere Antworten verlangen die Berücksichtigung eines größeren Rahmens. Hätte ein frühzeitiger und offener Meinungsaustausch zwischen Fraktion und Regierungsspitze die Zustimmung der Parlamentarier und der führenden Regierungspartei zur Agenda erleichtert? Oder wäre in diesem Fall bereits die Formulierung

78 Vgl. Karlheinz Niclauß, Kanzlerdemokratie, a.a.O. (Fn. 1), S. 356.

79 Reimut Zohlnhöfer, Zwischen Kooperation und Verweigerung, in: Christof Egle I ders. (Hrsg.), a.a.O. (Fn. 35), S. 124 - 150, S. 147 f.

80 Karl-Rudolf Korte, Der Pragmatiker des Augenblicks, in: Christof Egle / Reimut Zohlnhöfer (Hrsg.), a.a.O. (Fn. 35), S. $168-196$, S. 179 f. 
des Reformentwurfs und seine spätere Umsetzung auf unüberwindbare Schwierigkeiten gestoßen? Möglicherweise hätte eine intensivere Mitsteuerung der Fraktion das Reformkonzept auch inhaltlich verbessert und damit spätere Nachbesserungen und Revisionen überflüssig gemacht. All diese Überlegungen laufen auf die Frage hinaus: „Was wäre gewesen, wenn ...?"“. Diese Frage lässt sich jedoch bei einer Bewertung der Reformpolitik in der Regierungszeit Gerhard Schröders nicht vollständig vermeiden. 